\title{
Ambiguità delle sigle e questione di genere: il caso di Tgr
}

Ilaria Bonomi

PUBBLICATO: 12 MARZO 2021

\section{Quesito:}

Parecchi lettori hanno chiesto, alcuni lamentandosene, le ragioni e la liceità dell'espressione la $\operatorname{Tg} r$, pensandola riferita al telegiornale: qualcuno ha anche citato un improbabile la tg;, o addirittura la tg. E qualcuno ha addirittura erroneamente inserito anche questo 'strano' genere femminile nella tendenza generale a creare il femminile dei nomi di professione in modalità 'normali' per la nostra lingua, ma che sono molto discusse: una tendenza illustrata e argomentata da Vittorio Coletti nel tema di questo mese.

\section{Ambiguità delle sigle e questione di genere: il caso di $\operatorname{Tg} r$}

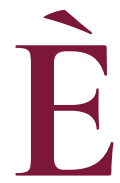

necessario subito fare chiarezza per evitare estensioni e fraintendimenti inopportuni.

Premessa fondamentale è l'esistenza della medesima sigla Tgr per indicare sia 'testata giornalistica regionale', sia 'telegiornale regionale'. Per essere più precisi, la sigla nasce come acronimo di testata giornalistica regionale nel I99I, in sostituzione della dicitura Rai regione, a indicare dal i987 la costola dei notiziari regionali scorporati dalla terza rete televisiva, a cui erano inizialmente legati.

Dunque, quando è usato con l'articolo femminile l'acronimo Tgr è riferito alla testata, mentre quando è usato nel senso di 'telegiornale' è preceduto regolarmente dall'articolo maschile. Questo appare chiaro e confermato da una breve ricerca svolta sull'archivio del "Corriere della Sera" e della "Repubblica", che riflette chiaramente la storia e l'uso della sigla Tgr (di solito con l'iniziale maiuscola), a partire dai primi anni Novanta del secolo scorso. Qualche esempio chiarificatore:

\section{Sedi regionali. Saranno chiusi 5 programmi. La Tgr insorge}

Oggi alle Ir e alle I5,30 Alberto Maccari, direttore del Tgr (il tg delle testate regionali Rai), riceverà il comitato di redazione, cioè il sindacato, delle redazioni di Napoli e di Milano. [...] Il malcontento è insomma notevole, nella più popolata testata della tv pubblica (ogni capoluogo di provincia ha una propria sede. Difatto la Tgr è la catena di trasmissione di una informazione locale capillare che sbocca nei notiziari televisivi e radiofonici locali). Perché questa protesta? Tutto è legato al varo del nuovo piano editoriale, cioè il timone della futura linea del Tgr secondo le intenzioni espresse da Maccari. ("Corriere della Sera", Io/3/2010)

Ieri il consiglio di amministrazione della Rai ha nominato i nuovi direttori di $\mathrm{Tg}_{1}$, $\mathrm{Tg}_{2}$ e Tg3, della $\mathrm{TgR}$ e del Gr radio. ("Corriere della Sera", I/II/20I8)

Spesso, poi, $\operatorname{la} \operatorname{Tg} r$ viene accostata, sempre intendendo la testata, al nome della relativa regione: $\operatorname{la} \operatorname{Tg} r$ Liguria, la Tgr Puglia, ecc.

A indicare il telegiornale regionale e non la testata appare talvolta, oltre alla sigla con l'articolo maschile, l'espressione, ovviamente sempre al maschile, Tg regionale. Ma va anche osservato che qualche volta, ma per fortuna raramente, l'uso della sigla al femminile può indurre a qualche ambiguità, potendo essere interpretato come riferito al telegiornale: 
Giovanni Minoli vorrebbe spostare i tg di mezza sera di un'ora in avanti. Se il $\operatorname{Tg} 3$ delle 22.30 e la Tgr delle 22.45 si facessero più in là, "la rete avrebbe una prima e seconda serata... " "la Repubblica", I7/7/1997)

Se, dunque, almeno stando alla documentazione scritta tratta dai quotidiani (ben più arduo sarebbe raccogliere una documentazione dai tg regionali trasmessi, che peraltro talvolta sembrano riferirsi alla sigla definendosi come 'telegiornali rai'), possiamo essere certi che la $\operatorname{Tg} r$ non è, salvo rarissimi casi, un'indebita estensione al femminile del corretto genere maschile per il telegiornale, regionale o nazionale che sia, chiudiamo notando, un po' scherzosamente, che la Tgr è stata usata anche per 'la tariffa per la gestione dei rifiuti', passata attraverso le più o meno trasparenti sigle TARSU 'tassa sui rifiuti solidi urbani', TGR appunto 'tariffa per la gestione dei rifiuti' (durata poco, per fortuna, certo anche per la sovrapposizione con la $\operatorname{tg} r$ giornalistica), TARI, tuttora in uso... fino alla prossima alternativa.

\section{Cita come:}

Ilaria Bonomi, Ambiguità delle sigle e questione di genere: il caso di Tgr , "Italiano digitale", 2021, XVI, 2021/1 (gennaio-marzo)

DOI: $10.35948 / 2532-9006 / 2021.5489$

\section{Copyright 2021 Accademia della Crusca}

Pubblicato con licenza creative commons CC BY-NC-ND 